\title{
Case for diagnosis. Suppurative nodule or something deeper?*
}

\author{
Nuno Preto Gomes ${ }^{1}$, Miguel Costa-Silva ${ }^{1}$, Elisabete Moreira ${ }^{1}$, Filomena Azevedo $^{1}$
}

DOI: http:/ / dx.doi.org/10.1590/abd1806-4841.20198675

\section{CASE REPORT}

A 62-year-old man with surgical history of umbilical hernioplasty seven years before and left total hip arthroplasty nine years previously presented with a 9-centimeter infiltrated brownish plaque on the posterior area of the left thigh with three months of evolution, centered by a 3-centimeter suppurative nodular lesion that emerged one week before (Figure 1A). The patient did not present systemic symptoms or elevated inflammatory markers. Exci- sional biopsy showed no signs of a neoplastic process. In the microbiological analysis, beta-lactam-sensitive Streptococcus agalactiae was isolated. Mycobacteriological and mycological tests were negative. Computed tomography of the left lower limb revealed a 12-centimeter linear extension of the lesion to the posterior side of the proximal left femur (Figure 1B). The patient was referred to orthopedics for revision of the prosthesis.
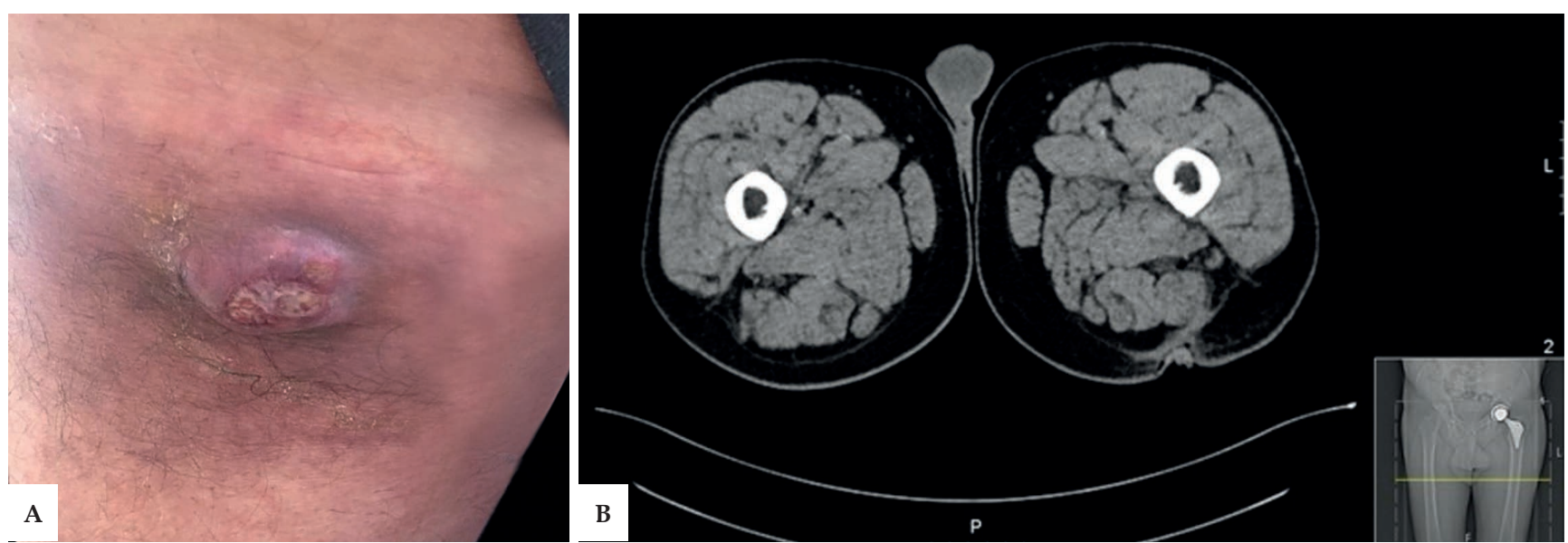

FIGURE 1: A - Infiltrated plaque in the posterior area of the left thigh centered by a suppurative nodular lesion B - Computed tomography of the left lower limb showing the 12-centimeter linear path to the posterior part of the left proximal femur

Received 05 August 2018.

Accepted 22 October 2018

* Work conducted at the Centro Hospitalar São João, Porto, Portugal.

Financial support: None.

Conflict of interest: None.

1 Dermatology and Venereology Service, Centro Hospitalar São João, Porto, Portugal.

MAILING AdDRESS:

Nuno Miguel Preto Gomes

E-mail: nunompretogomes@gmail.com

C2019 by Anais Brasileiros de Dermatologia 


\section{DISCUSSION}

Total hip arthroplasty (THA) is one of the most successful procedures in the history of orthopedic surgery. ${ }^{1}$ Although prosthesis joint infection (PJI) is rare, it causes significant morbidity and can occur not only during surgery but throughout the implant's lifetime. It can be classified as early, delayed, or late (more than 24 months after surgery), as in this case. While early and delayed infections are usually acquired during implantation of the prosthesis, late infections are predominantly acquired by hematogenous seeding. The most frequent sources of bacteremia are skin, respiratory tract, dental, and urinary tract infections. ${ }^{2}$

One of the accepted criteria for diagnosis of joint infections is a sinus tract communicating with the joint, as occurred in this case. ${ }^{1}$ Hip PJI is most commonly caused by coagulase-negative staphylococci or Staphylococcus aureus. Several modifiable and non-modifiable factors are associated with the long-term risk of developing THA infection, such as smoking, obesity, diabetes, depression, steroid use, and frailty. ${ }^{3,4}$ Identification of modifiable factors and their beneficial modulation can be effective in reducing the incidence of PJI. Successful treatment of PJI depends on extensive surgical debridement and adequate and effective antibiotic therapy. Treatment in two stages using a spacer is recommended for most chronic PJI. ${ }^{5}$ In the current case, resolution of the lesion occurred after prosthesis revision surgery and subsequent focus control.

The present case calls attention to the possibility of cutaneous fistulization of prosthetic infections, even if the surgery was performed several years before. Although the diagnosis and treatment of THA infection have improved in the last decade, the entity can mimic a neoplastic process or another infectious disease, so the physician's level of suspicion should be high. The complexity of treatment makes early diagnosis even more essential. $\square$

\begin{abstract}
The authors report the case of a 62-year-old man with a history of total left hip arthroplasty nine years previously presenting with a large infiltrated plaque on the posterior area of the left thigh with three months of evolution without systemic symptoms or elevated inflammatory markers. Computed tomography of the left lower limb revealed a 12-centimeter linear extension of the lesion to the posterior part of the left proximal femur. Prosthesis joint infection, although rare, is a surgical complication to be taken into account, even if the surgery was performed many years before.
\end{abstract}

Keywords: Arthroplasty, replacement, hip; Cutaneous fistula; Skin abnormalities; Skin manifestations

\section{REFERENCES}

1. Parvizi J, Fassihi SC, Enayatollahi MA. Diagnosis of Periprosthetic Joint Infection Following Hip and Knee Arthroplasty. Orthop Clin North Am. 2016;47:505-15

2. Zimmerli W, Trampuz A, Ochsner PE. Prosthetic-joint infections. N Engl J Med. 2004;351:1645-54.

3. Lenguerrand E, Whitehouse MR, Beswick AD, Kunutsor SK, Burston B, Porter $M$, et al. Risk factors associated with revision for prosthetic joint infection after hip replacement: a prospective observational cohort study. Lancet Infect Dis. 2018;18:1004-1014.
4. Kunutsor SK, Whitehouse MR, Blom AW, Beswick AD; INFORM Team. PatientRelated Risk Factors for Periprosthetic Joint Infection after Total Joint Arthroplasty: A Systematic Review and Meta-Analysis. PLoS One. 2016;11:e0150866.

5. Lee HD, Prashant K, Shon WY. Management of Periprosthetic Hip Joint Infection. Hip Pelvis. 2015;27:63-71.

\footnotetext{
AUTHORS'CONTRIBUTIONS

Nuno Preto Gomes (iD) ORCID

0000-0003-4278-3106

Statistical analysis; Approval of the final version of the manuscript; Conception and planning of the study; Elaboration and writing of the manuscript; Obtaining, analyzing and interpreting the data; Effective participation in research orientation; Intellectual participation in propaedeutic and/or therapeutic conduct of the cases studied; Critical review of the literature; Critical review of the manuscript

Miguel Costa-Silva ORCID 0000-0003-4012-5660

Statistical analysis; Approval of the final version of the manuscript; Conception and planning of the study; Elaboration and writing of the manuscript; Obtaining, analyzing and interpreting the data; Effective participation in research orientation; Intellectual participation in propaedeutic and/or therapeutic conduct of the cases studied; Critical review of the literature; Critical review of the manuscript

Elisabete Moreira

0000-0001-7311-0145

Approval of the final version of the manuscript; Elaboration and writing of the manuscript; Effective participation in research orientation; Intellectual participation in propaedeutic and/ or therapeutic conduct of the cases studied; Critical review of the literature

Filomena Azevedo $\quad$ (iD) ORCID 0000-0003-0402-6382

Approval of the final version of the manuscript; Elaboration and writing of the manuscript; Intellectual participation in propaedeutic and/or therapeutic conduct of the cases studied; Critical review of the literature
}

How to cite this article: Preto Gomes N, Costa-Silva M, Moreira E, Azevedo F. Case for diagnosis. Suppurative nodule or something deeper? An Bras Dermatol. 2019;94(3):363-4. 